Authors

Mark Notcutt, Long-Sheng Ma, Andrew D. Ludlow, Seth M. Foreman, Jun Ye, and John L. Hall 


\title{
Contribution of thermal noise to frequency stability of rigid optical cavity via Hertz-linewidth lasers
}

\author{
Mark Notcutt, ${ }^{*}$ Long-Sheng Ma, ${ }^{\dagger}$ Andrew D. Ludlow, ${ }^{\ddagger}$ Seth M. Foreman, ${ }^{\ddagger}$ Jun Ye, ${ }^{\ddagger}$ and John L. Hall ${ }^{\ddagger}$ \\ JILA, University of Colorado, Boulder, Colorado 80309, USA \\ and National Institute of Standards and Technology, Boulder, Colorado 80309, USA
}

(Received 22 July 2005; published 21 March 2006)

\begin{abstract}
We perform detailed studies of state-of-the-art laser stabilization to high finesse optical cavities, revealing fundamental mechanical thermal noise-related length fluctuations. We compare the frequency noise of lasers tightly locked to the resonances of a variety of rigid Fabry-Perot cavities of differing lengths and mirror substrate materials. The results are in agreement with the theoretical model proposed in K. Numata, A. Kemery, and J. Camp [Phys. Rev. Lett. 93, 250602 (2004)]. The results presented here on the fundamental limits of FP references will impact planning and construction of next generation ultrastable optical cavities.
\end{abstract}

DOI: 10.1103/PhysRevA.73.031804

PACS number(s): 42.60.Da, 05.40.Jc, 42.62.Eh

Fabry-Perot (FP) cavities with resonance full width half maximums of $\sim 10 \mathrm{kHz}$ and exceptional length stability are essential tools in the frequency stabilization of lasers for precision spectroscopy [1], optical atomic clocks [2,3], quantum optics experiments, and interferometric gravity wave detectors [4]. The most common limits to the frequency stability of a cavity stabilized laser are photon shot noise, residual amplitude modulation (RAM) contaminating the error signal, and changes of the cavity length from accelerations. The latter limitation has been overcome with vibration isolation of Ref. [1] and the use of a mounting geometry that reduces acceleration sensitivity [5], resulting in linewidths typically less than $1 \mathrm{~Hz}$, with the best system being $0.16 \mathrm{~Hz}$ [1]. Recently, a theoretical formulation of thermal noise driven displacement fluctuations in rigid FP cavities [6] has proposed that such fluctuations may limit the length (and thus frequency) stability of these frequency references. Indeed, these theoretical limits [6], which are of the order of 1 $\times 10^{-16} \mathrm{~m} / \sqrt{\mathrm{Hz}}$ at $1 \mathrm{~Hz}$ as a displacement (for a ULE [12] (Corning 7972 Ultra Low Expansion) cavity of $\sim 20 \mathrm{~cm}$ length), appear to be able to explain two previously published stability results $[1,4]$. Here we present the first systematic and detailed experimental confirmation of this thermal noise model. The thermal noise fluctuations of suspended mirrors for interferometric gravity wave detectors have been measured in a test experiment [7] using short cavities. Because of their typical $\sim 10 \mathrm{~cm}$ lengths and the $1 / f$ power spectral density of the displacement fluctuations in mechanical systems with internal damping [8-10], thermal noise is an observable effect in rigid cavities at Fourier frequencies of $\sim 10 \mathrm{~Hz}$ or less. The thermal noise limits to the frequency stability of Fabry-Perot cavities are problematic for future

\footnotetext{
*Present address: Lockheed Martin Coherent Technologies, Louisville, CO; electronic address: notcutt@jilau1.colorado.edu

${ }^{\dagger}$ Also affiliated with BIPM, Sevres, France, and East China Normal University, Shanghai.

${ }^{\ddagger}$ Also affiliated with Department of Physics, University of Colorado, Boulder, CO, 80309.
}

optical standards, many of which are based on optical transitions tens of $\mathrm{mHz}$ in linewidth [2].

In this Rapid Communication, we present a detailed and systematic experimental investigation of thermal-noisedriven length fluctuations in various Fabry-Perot cavities over the Fourier frequency range of 0.03 to $100 \mathrm{~Hz}$, confirming the model proposed in [6]. This was achieved by measuring the frequency noise of a laser whose frequency was tightly servo-locked to a resonance of the cavity, and comparing the cavity-locked laser with a second independent laser, frequency-stabilized to its own cavity, that had a stability comparable with or better than the one under test [5].

From the fluctuation-dissipation theorem, the most significant parameters in the calculation of the thermal noise spectrum for a given structure are the mechanical quality $(Q)$ factor and Young's modulus of the material [8]. From the "order-of magnitude" formulas given in [6], the power spectral density of mirror thermal noise displacement (which typically dominates the spacer contribution) is

$$
G(f)=\frac{4 k_{B} T}{\omega} \frac{1-\sigma^{2}}{\sqrt{\pi} E w_{0}} \phi_{\text {sub }}\left(1+\frac{2}{\sqrt{\pi}} \frac{1-2 \sigma}{1-\sigma} \frac{\phi_{\text {coat }}}{\phi_{\text {sub }}} \frac{d}{w_{0}}\right),
$$

where $k_{B}$ is Boltzmann's constant, $T$ is the temperature, $\sigma$ is Poisson's ratio, $E$ is the Young's modulus, $w_{0}$ is the beam radius, $\phi=1 / Q$, the loss angle of the substrate or coating (as labeled), and $d$ is the coating thickness $(\sim 5 \mu \mathrm{m})$. The term preceding the bracket is the contribution of the substrate while the term inside the bracket is the modifying effect of the coating. In our experiments, the materials from which the cavities are made are all glasses with similar Young's modulus. However, an important quantity, the mechanical $Q$ factors of the different materials, spans a factor of 300 in different cases. A comparison of noise from cavities using identical mirrors with spacers of different length, tested whether the mirror noise dominated the spacer noise. Lastly, the noise of a $\mathrm{TEM}_{00}$ mode and a $\mathrm{TEM}_{24}$ mode were compared to test the dependence on mode radius $w_{0}$.

For all the measurements described here, the frequency reference was a Nd:YAG laser at $1064 \mathrm{~nm}$ frequency locked to a resonance of a Fabry-Perot cavity made of ULE (Corn- 
TABLE I. Properties of cavities tested for frequency noise. Zer $=$ Zerodur [12] and FS $=$ Fused silica and the loss angle $\phi$ of the materials [6]. The coating loss angle is $4 \times 10^{-4}[6,11]$. The dimensions of the spacer are given in columns 4 and 5 , and column 6 shows the approximate mode spot sizes (intensity $1 / e^{2}$ radius) on the cavity mirrors. The Poisson ratio is 0.17 (ULE and FS), and 0.24 (Zer). The Young's modulus is $\sim 67 \times 10^{9} \mathrm{~Pa}$ for ULE, Zer, and FS.

\begin{tabular}{|c|c|c|c|c|c|}
\hline Case & $\begin{array}{l}\text { Spacer } \\
\text { material }\end{array}$ & $\begin{array}{l}\text { Mirror substrate } \\
\text { material and loss } \\
\text { angles }[8]\end{array}$ & $\begin{array}{l}\text { Spacer } \\
\text { length } \\
(\mathrm{mm})\end{array}$ & $\begin{array}{l}\text { Spacer } \\
\text { radius } \\
(\mathrm{mm})\end{array}$ & $\begin{array}{l}\text { Mode } \\
\text { size } \\
(\mu \mathrm{m})\end{array}$ \\
\hline Reference cavity & ULE & $\operatorname{ULE}\left(\phi=1.6 \times 10^{-5}\right)$ & 50 & 6.3 & 175 \\
\hline $\mathrm{A}$ & Zer & $\mathrm{FS}\left(\phi=1 \times 10^{-6}\right)$ & 35 & 15 & 250 \\
\hline $\mathrm{B}$ & Zer & $\operatorname{Zer}\left(\phi=3 \times 10^{-4}\right)$ & 35 & 15 & 100 \\
\hline B2 $\mathrm{TEM}_{24}$ & Zer & Zer & 35 & 15 & 170 \\
\hline C $698 \mathrm{~nm}$ & Zer & Zer & 35 & 15 & 175 \\
\hline $\mathrm{D}$ & Zer & Zer & 10 & 12.5 & 130 \\
\hline $\mathrm{E}$ & Zer & FS & 10 & 12.5 & 185 \\
\hline
\end{tabular}

ing 7972) [12], a system that had previously demonstrated sub-Hz linewidths [5]. With typically $100 \mu \mathrm{W}$ incident on the cavity, shot-noise-limited locking contributed a frequency noise linear spectral density of $0.1 \mathrm{~Hz} / \sqrt{\mathrm{Hz}}$. The optical system of the cavity under test was very similar to the reference laser system. Cavities of different geometries were swapped out from the mounting structure, with other parts of the mechanical, optical, and electronic systems remaining largely unchanged. Light was transferred through an optical fiber (with a phase noise cancellation servo) from the reference laser system to the isolated platform of the system under test [13], where a heterodyne beat between the light from the two lasers was detected on a photodiode. A third laser system was constructed at $698 \mathrm{~nm}$ [2] and the frequency instability of this laser was measured [14] with our reference laser and a broadband, frequency stabilized, femtosecond comb.

We discuss the results in three sections corresponding to tests of the noise dependence on mirror material $Q$ factor, length, and mode radius. The properties of the reference cavity and the six cavities under test are listed in Table I. The results of the beat frequency instability analysis for these cavities is shown in Fig. 1. Table II summarizes the results shown in Fig. 1 and compares the resulting experimental data with values calculated from the order-of-magnitude formulas in [6].

The mechanical loss of the cavity mirrors was varied by replacement of fused silica (FS) substrate mirrors [A in Fig. 1(b) and Table I] with Zerodur substrate mirrors (B). The high $Q$ factor of the fused silica substrate is significantly modified by the effect of the coating (400 times lower in $Q$ factor) though scaled by $d / w_{0} \sim 1 / 35$, in the power spectral density in Eq. (1).

The optically contacted FS mirrors were removed from the spacer, the second Zerodur mirror set (with similar contrast and finesse to the first) contacted to the same spacer, and the altered cavity returned to the original mounting structure. The optical and electronic systems remained unchanged except for a small change in the proportional gain of the loop filter for servo-locking. Curves A and B of Fig. 1(b) show the Allan deviation of the heterodyne beat of the stabilized reference laser and the lasers locked to cavities la- beled A and B in Table I, respectively. The $\sim 100 \mathrm{MHz}$ beat signal data were analyzed by a frequency counter with typically four sets of $1000 \mathrm{~s}$ long records per curve plotted here. The data possessed a linear frequency drift ranging from typical values of $10 \mathrm{~Hz} / \mathrm{s}$ (cavity length creep) to an extreme case of $150 \mathrm{~Hz} / \mathrm{s}$ for one of the short cavities. In this case, inadequate spacer surface flatness prevented good optical contacts and the spacer was not chemically etched to remove surface damage from machining. These drifts were removed in data processing by subtracting off the linear frequency drift from the time series data. Nonlinear components of the drift, due to temperature changes of the cavity, had periods of many hours. The residual from each fit was then analyzed to give the Allan deviation. Curves A and B can be compared at an integration time of $\sim 1 \mathrm{~s}$ to give a stability ratio of 4.6, corresponding closely to the ratio from the calculated values. Curves AS and BS in Fig. 1(a) show the more traditional spectral density data for cavities A and B. Although the spectral nature of the stability curve is slightly different to the theoretical $1 / \sqrt{f}$ as a displacement, the ratio of the quantities agrees with Eq. (1).

The length of the cavity was changed by switching the same Zerodur substrate mirrors from the $35 \mathrm{~mm}$ long Zerodur spacer [B in Fig. 1(b) and Table I] to a $10 \mathrm{~mm}$ long Zerodur spacer (D). The short cavity spacer was not mounted with the same degree of lengthwise symmetry, as in cases A and $\mathrm{B}$, hence there is an increase in acceleration-related noise visible at $0.02 \mathrm{~s}$ and in spectrum DS. Although curves B and $\mathrm{D}$ differ somewhat in their slopes, the ratio of the Allan deviations of curve $\mathrm{D} / \mathrm{B}$ is close to the inverse ratio of their lengths, 3.5, indicating that mirror substrate thermal noise fluctuations dominate spacer-length fluctuations.

For different mode radii, the Allan deviation of the laser locked to the $\mathrm{TEM}_{00}$ mode and $\mathrm{TEM}_{24}$ mode of the same cavity are shown in Fig. 1(c) as curves B and B2. Angular fluctuations of the cavity mode and input beam caused the bump in curve B2: however, a comparison of the variance curves B and B2 at $8 \mathrm{~s}$ integration time gives a ratio of 1.4, which is in close agreement with the square root of the ratio of the mode diameters, 1.3.

Curves $\mathrm{B}$ and $\mathrm{C}$ show the difference between cavities of the same dimensions and material (though with different mir- 


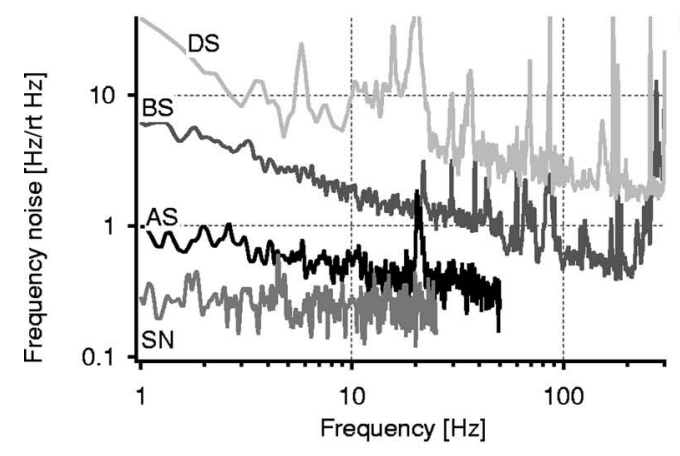

(a)

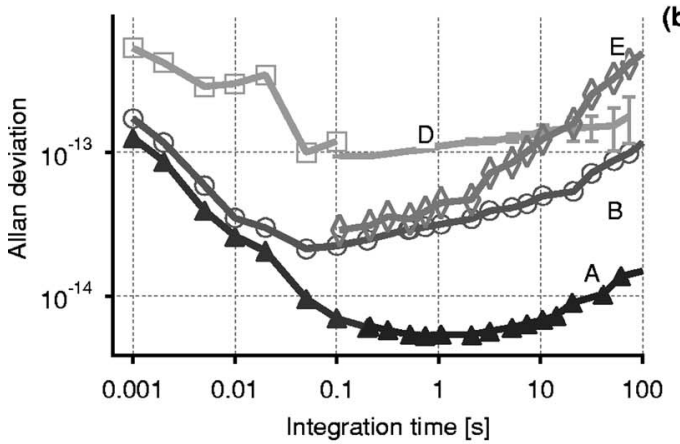

(b)

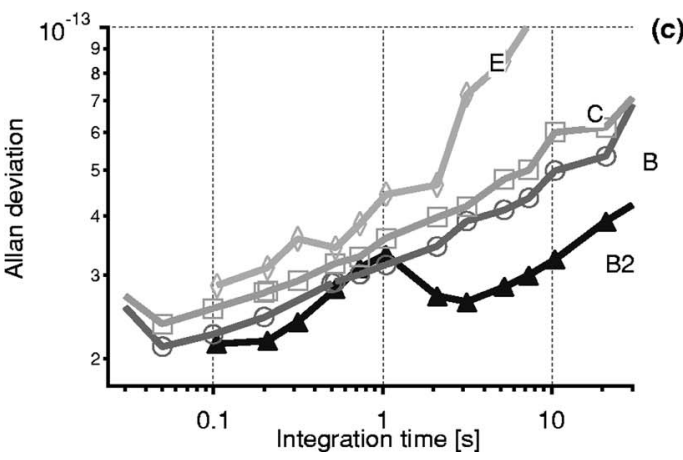

FIG. 1. (a) Single sided frequency noise spectral density data for the beats of the reference laser vs the cases A (AS), B (BS), and D (DS) of Table I. Curve SN is the spectrum, in frequency units, of the error signal when not resonant with the cavity for cases A and B. (b) Allan deviation data for the beats of the reference laser vs cases A, B, D, and E. Typical error bars are shown for the Allan deviation of curve D. (c) Allan deviation data for the beats of the reference laser vs cases B, B2, C, and E.

ror curvatures) at two wavelengths, $698 \mathrm{~nm}(\mathrm{C})$ and $1064 \mathrm{~nm}$ (B). Although a third, markedly different laser system with different noise and modulation properties was used with cavity $\mathrm{C}$, the resulting stability is similar to the $1064 \mathrm{~nm}$ cavities, giving independent confirmation of our results.

We now consider noise contributions. To estimate the total instability of the reference cavity, including locking error, thermal noise and thermal expansion, the order-of-magnitude calculations [6] indicate that the beat frequency instability [curve A of Fig. 1(b)], has approximately equal contributions from the reference laser and the cavity under test. The instability of the reference laser may therefore be estimated as curve A divided by $\sqrt{2}$. The noise floor of the locking to each cavity is the combination of shot noise, RAM, and at times greater than $3 \mathrm{~h}$, temperature change of the cavities. At Fourier frequencies less than $5 \mathrm{~Hz}$, RAM is the largest source of locking error. Because RAM has several possible origins (e.g., parasitic etalons and geometrical effects), we measured the net noise floor of locking the laser to the cavity by tuning the laser away from any detectable cavity resonance, recording the error signal and converting it to frequency units with the measured error signal slope. This quantity was scaled by the cavity contrast to account for the power level detected when the laser is locked to the cavity. The noise floor at Fourier frequencies $>15 \mathrm{~Hz}$ is close to the calculated noise density for shot noise for our experimental parameters. The contribution of RAM and shot noise to the beat is plotted in curve SN of Fig. 1(a), and is clearly distinguishable from the best frequency noise spectral density of cavity A, curve AS.

Column 4 of Table II is the ratio between the lowest measured value and the calculated Allan deviation. Comparison of the magnitudes of expected and measured noise is complicated because of the difference in the slopes of their Allan deviations. The increase of a factor of $\sim 2.3$ between the measured and theoretical values may be attributed to the low mechanical $Q$-factor of the cavity assembly due to the use of glue or silicone sealant adhesives [5] and a support structure of Teflon. The adopted value of $\phi_{\text {coat }}$ of $4 \times 10^{-4}$ [6] is also one of the best on record and may not be representative of our unmeasured mirrors.

Theory [6] predicts a flat line of Allan deviation $(1 / f$ power spectral density), while power-law curve fits to the three best data sets [curves B, C, and D of Figs. 1(b) and 1 (c) $]$ are $\sim \tau^{0.25}$ in the range of integration times of 0.1 to $30 \mathrm{~s}$, corresponding to power spectral densities of $\sim f^{-1.25}$. Since the cavity of case A has a higher thermal expansion coefficient of its effective length (because of its fused silica mirrors) than others and because the temperature controllers were left running unaltered over the length of the experiment, thermal expansion therefore contributes little to the slopes of curves B, C, and D. The frequency dependence of the loss angle of materials such as silica have a power-law relationship of $\sim f^{0.8}[11]$, which has the wrong frequency dependence to account for the difference here between theory and measurement. Thermoelastic noise can be excluded as a noise source for the cavities used here [6]. In other experiments of the measurement of off-resonance thermal noise $[7,9]$, the fit is excellent. We note that our measurements are at frequencies that are significantly lower than $[7,9]$ as a fraction of the first mechanical resonance frequency, $\sim 1 \mathrm{~Hz} / 123 \mathrm{kHz}$. Without a quantitative explanation for the frequency dependence of our data, we note that the properties of optical contacts between the mirrors and spacer are variable and the contact could degrade the $Q$ factor of the mirror substrate or add displacement noise of its own [6]. As mentioned, the $Q$ factor of the cavity may have a frequency dependence, because of the glues and sealants and Teflon used in the construction and supports.

In conclusion, we provide the most systematic and convincing measurements to date of the thermal-noise-driven length fluctuations of a range of Fabry-Perot cavities. Results are comparable with theory. With the mirrors contributing the largest length fluctuations, due to the loss angle of the coatings, the optimal length of future FP cavities may be a compromise between thermal noise and acceleration sensitivity considerations, as the acceleration sensitivity of a cav- 
TABLE II. Comparison of theory and measurements of thermal noise displacement fluctuations expressed as Allan deviation (A-dev), including the ratio of measured/calculated values for the cavities listed in Table I. Also included is the spectral density value of equivalent cavity length fluctuation at $1 \mathrm{~Hz}$.

\begin{tabular}{lcccc}
\hline \hline \multicolumn{1}{c}{ Case } & $\begin{array}{c}\text { Calculated } \\
\text { A-dev } \\
\text { of beat }\end{array}$ & $\begin{array}{c}\text { Measured } \\
\text { lowest } \\
\text { point A-dev }\end{array}$ & $\begin{array}{c}\text { Ratio } \\
\text { measured/calc }\end{array}$ & $\begin{array}{c}\text { Measured } \\
\text { displacement noise } \\
\mathrm{m} / \sqrt{\mathrm{Hz}}\end{array}$ \\
\hline $\begin{array}{l}\text { Reference } \\
\text { cavity } \\
\text { only }\end{array}$ & $1.8 \times 10^{-15}$ & & & \\
A & & & & \\
B & $2.8 \times 10^{-15}$ & $5.0 \times 10^{-15}$ & 1.8 & $1.7 \times 10^{-16}$ \\
B2 & $1.3 \times 10^{-14}$ & $2.0 \times 10^{-14}$ & 1.6 & $1.0 \times 10^{-15}$ \\
C & $9.4 \times 10^{-15}$ & & & \\
D & $9.4 \times 10^{-15}$ & $2.5 \times 10^{-14}$ & 2.7 & $1.2 \times 10^{-15}$ \\
E & $3.8 \times 10^{-14}$ & $1.0 \times 10^{-13}$ & 2.7 & $2.7 \times 10^{-16}$ \\
\hline \hline
\end{tabular}

ity increases with length [5]. Designs that have common mode rejection of acceleration may become important [5]. The reduction of mechanical losses in dielectric coatings is currently under investigation [11]. Using fused silica mirror substrates and a ULE spacer is one compromise, although the thermal expansion of the cavity can be expected to be $\sim 10^{-7} \mathrm{~K}^{-1}$ which is large compared with ULE $\left(\sim 10^{-9} \mathrm{~K}^{-1}\right)$. Using a mode of large radius is possible through high order off-axis transverse cavity modes, although the necessary mode matching is more complex. Cryogenic cooling would provide improvement scaling with the square root of the temperature, though disappointingly the $Q$ factor of the coatings does not increase with reduced temperature [15]. Using a hybrid cavity/atomic or molecular reference for times $>1 \mathrm{~s}$ is certainly a possibility [16] although less flexible than only a cavity.

We are indebted to R. Lalezari of Advanced Thin Films for coatings and optical contacting and to the Electronics and Instrument shops at JILA. We acknowledge useful discussions with K. Numata, J. Bergquist, L. Hollberg, and C. Oates. Funding for this work was provided by the ONR, NASA, NIST, and NSF.
[1] B. C. Young et al., Phys. Rev. Lett. 82, 3799 (1999).

[2] A. D. Ludlow, M. M. Boyd, T. Zelevinsky, S. M. Foreman, S. Blatt, M. Notcutt, T. Ido, and J. Ye, Phys. Rev. Lett. 96, 033033 (2006).

[3] Frequency Measurement and Control: Advanced Techniques and Future Trends, edited by A. N. Luiten (Springer Verlag, Berlin, 2001).

[4] F. Bondu et al., Opt. Lett. 21, 582 (1996).

[5] M. Notcutt et al., Opt. Lett. 30, 1815 (2005).

[6] K. Numata, A. Kemery, and J. Camp, Phys. Rev. Lett. 93, 250602 (2004).

[7] K. Numata, M. Ando, K. Yamamoto, S. Otsuka, and K. Tsub- ono, Phys. Rev. Lett. 91, 260602 (2003).

[8] P. R. Saulson, Phys. Rev. D 42, 2437 (1990).

[9] G. I. Gonzalez and P. R. Saulson, Phys. Lett. A 201, 12 (1995).

[10] C. C. Speake et al., Meas. Sci. Technol. 10, 430 (1999).

[11] D. R. M. Crooks et al., Class. Quantum Grav. 21, S1059 (2004).

[12] Use of the trade name is for informational purposes only.

[13] L.-S. Ma et al., Opt. Lett. 19, 1777 (1994).

[14] L. Hollberg et al., Metrologia 42, S105 (2005).

[15] K. Yamamoto et al., Class. Quantum Grav. 21, S1075 (2004).

[16] C. Ishibashi et al., Proc. SPIE 4634, 58 (2002). 